\title{
Fatores de riscos intrínsecos e extrínsecos que predispõe ao delirium em pacientes internados em Unidade de Terapia Intensiva: revisão integrativa
}

Intrinsic and extrinsic risk factors that predispose to delirium in patients hospitalized in Intensive Care Unit: integrative review

Factores de riesgo intrínsecos y extrínsecos que predeciran al delirio en pacientes ingresados a la Unidad de Terapia Intensivo: revisión integradora

Cristiane Martins Serra Pires ORCID: https://orcid.org/0000-0002-0015-4021 Hospital São Domingos, Brasil

Mara Ellen Silva Lima

ORCID: https://orcid.org/0000-0002-9016-8143 Hospital São Domingos, Brasil

E-mail: maraellensl@ hotmail.com

Maria Cristiane Aranha Brito ORCID: https://orcid.org/0000-0002-6979-8773 Centro Universitário Maurício de Nassau, Brasil E-mail: tiane91@hotmail.com

Luciana Patrícia Lima Alves Pereira ORCID: https://orcid.org/0000-0002-6285-4330

Universidade Estadual do Maranhão, Brasil

E-mail: llucianapatricia@yahoo.com.br

Ana Paula Muniz Serejo

ORCID: https://orcid.org/0000-0002-4376-4364 Centro Universitário Maurício de Nassau, Brasil

E-mail: apsmuniz1@gmail.com

Marjanne Pestana Ferreira ORCID: https://orcid.org/0000-0001-8314-7857 Instituto de Ensino Superior Franciscano, Brasil

E-mail: marjanne_s@hotmail.com

Julianne Lima da Conceição ORCID: https://orcid.org/0000-0002-1470-0169 Instituto de Ensino Superior Franciscano, Brasil E-mail: julianne-lima@hotmail.com

Josilene Sousa Bastos ORCID: https://orcid.org/0000-0003-3581-6053 Instituto de Ensino Superior Franciscano, Brasil E-mail: josilenysousa@yahoo.com 
Paulo Henrique Soares Miranda ORCID: https://orcid.org/0000-0001-9091-1059 Instituto de Ensino Superior Franciscano, Brasil E-mail: paulohenriquesoaresmiranda0@gmail.com Kássia Cristhine Nogueira Gusmão ORCID: https://orcid.org/0000-0002-1582-3232 Instituto de Ensino Superior Franciscano, Brasil E-mail: ksiagusmao@hotmail.com

\begin{abstract}
Resumo
O significado da palavra delirium está atribuído ao latim "delirare”, que significa "estar fora do lugar". Sobre os artigos incluídos nesta revisão, observou-se que houve uma prevalência de estudos publicados nos anos de 2016 e 2020. Como resultado, após a análise significativa do conteúdo, obteve os seguintes fatores que predispõe; idade igual ou maior que 60 anos, o uso de sedoanalgésicos, ventilação mecânica invasiva, restrição física, desidratação e desnutrição, ambiente típico de uma UTI e perturbação do sono. Diante deste estudo, podemos afirmar que o delirium é um problema muito comum em pacientes internados em UTI. O primeiro passo para intervir nessa situação é prevenir, criando protocolos de prevenção de delirium baseado nos fatores de risco com intuito de chamar a atenção dos profissionais para os pacientes que apresentarem um resultado positivo para esses fatores. deve ser considerado uma emergência na UTI, e educar os profissionais quanto a isso é de extrema importância, para que os mesmos fiquem em constante vigilância neurológica para que possam agir de forma assertiva no diagnóstico e tratamento do mesmo.
\end{abstract}

Palavras-chave: Delirium; Fatores de risco; Unidade de Terapia Intensiva.

\begin{abstract}
The meaning of the word delirium is attributed to the Latin "delirare", which means "to be out of place." On the articles included in this review, it was observed that there was a prevalence of studies published in the years of 2016 and 2020. As a result, after significant content analysis, he obtained the following predisposing factors: age equal to or greater than 60 years, the use of sedoanalgesics, invasive mechanical ventilation, physical restriction, dehydration and malnutrition, typical ICU environment and sleep disturbance. Given this study, we can say that delirium is a very common problem in patients admitted to the ICU. The first step to intervene in this situation is to prevent, creating delirium prevention protocols based on risk factors in order to call professionals' attention to patients who have a positive result for these factors. should be considered an emergency in the ICU, and educate professionals about it it is of utmost importance, so that they remain under constant neurological surveillance so that they can act assertively in the diagnosis and treatment of the same.
\end{abstract}

Keywords: Delirium; Risk factors; Intensive Care Unit.

\title{
Resumen
}

El significado de la palabra delirium se atribuye al latín "delirare", que significa "estar fuera de lugar". Sobre los artículos incluidos en esta revisión, se observó que hubo una prevalencia de estudios publicados en 2016 y 2020. Como como resultado, luego del análisis de contenido significativo se obtuvieron los siguientes factores predisponentes: edad igual o mayor a 60 años, uso de analgésicos sedantes, ventilación mecánica invasiva, restricción física, deshidratación y desnutrición, ambiente típico de una UCI y alteración del sueño. Podemos decir que el delirio es un problema muy común en los pacientes de UCI, el primer paso para intervenir en esta situación es prevenirlo, creando protocolos de prevención del delirio basados en factores de riesgo con el fin de llamar la atención de los profesionales hacia los pacientes que presentan un resultado positivo. Por estos factores, debe considerarse una emergencia en la UCI, y educar a los profesionales sobre esto es de suma importancia, p para que estén bajo constante vigilancia neurológica para que puedan actuar de manera asertiva en el diagnóstico y tratamiento de los mismos.

Palabras clave: Delirio; Factores de risco; Unidade de Cuidados Intensivos.

\section{Introdução}

De acordo com Gati (2017), o significado da palavra delirium está atribuído ao latim “delirare”, que significa "estar fora do lugar". De forma mais abrangente, Faustinol (2016) afirma que, o delirium é uma disfunção cerebral aguda potencialmente reversível, ou seja, uma perturbação no nível de consciência, cujos sintomas se iniciam de forma súbita, caracterizado por deixar os pacientes incapazes de prestarem atenção e pensarem com clareza em fatos novos, além da desorientação e flutuações. 
Delirium e delírio são terminologias semelhantes, porém com o significado, clínicae tratamento diferentes. O delírio trata - se de um sintoma oriundo de transtornos psiquiátricos, ocorrendo alteração do juízo de realidade em decorrência de pensamento patológico (Luz, 2016).

Os mecanismos fisiopatológicos do delirium ainda não estão totalmente esclarecidos. Porém o estudo de Bahia (2016) relata que há um aumento significativo e em raras vezes a diminuição de determinados neurotransmissores (acetilcolina, serotonina, dopamina, noradrenalina e GABA) no organismo, modulando o controle da função cognitiva, comportamento e humor.

Clinicamente, a literatura descreve três tipos de delirium, o hiperativo, em que há agitação e o aumento da atividade psicomotora, com tentativas de remoção dos dispositivos médicos; o tipo hipoativo onde ocorre a lentificação psicomotora, letargia, apatia e pouca resposta ou nenhuma aos estímulos externos e o misto, que tem como característica a flutuação imprevisível de sintomas entre os dois tipos descritos acima (Krebs, 2018).

Além dessa classificação, alguns autores relatam delirium subsindromático, em que existe a presença de alguns sintomas da disfunção, porém não cumpre o quadro completo paraos critérios do diagnóstico, porque os sinais se apresentam em menor número, ou seja, os doentes têm de um a três sinais (Bastos et al., 2019).

Para o diagnóstico do delirium, existem diversas escalas, como a Delirium Detection Score (DDS), Cognitive Test of Delirium (CTD), Memorial Delirium Assessment Scale (MDAS), Intensive Care Delirium Screening Checklist (ICDSC), The Neelon and Champagne Confusion Scale (NEECHAM), Delirium Rating Scale-Revised-98 (DRS-98-R), PREdiction of DELIRium in ICU patients (PRE-DELIRIC), porém na prática clínica a mais utilizada pelos intensivistas é a escala Confusion Assessment Method for the Intensive Care Unit (CAM-ICU), uma escala baseada nos critérios do DSM-IV, pois é um dos instrumentos de avaliação mais fidedigno para o diagnóstico do delirium (Lima, 2016).

A avaliação do delirium com o CAM-ICU, é aplicada quando ocorre alteração no estado mental de base do paciente e deve ser embasado no RASS (Richmond Agitation SedationScale) com pontuação +4 à - 3. Sistematicamente a aplicação desta escala de delirium se baseiaem 4 etapas que estimam a flutuação do nível de consciência. As etapas devem ser seguidas na ordem e o avaliador deverá ter habilidade para aplicá-la (Pereira, 2019).

Existem fatores de riscos intrínsecos e extrínsecos, onde o primeiro está relacionadoao paciente e que podem levar o indivíduo desenvolver o delirium e o outro está relacionado aos fatores do ambiente, que facilitam a desenvolver o problema dentro dessas unidades (Veiga, 2021).

Prayce (2019) relata que o tratamento pode ser realizado de forma farmacológica e não farmacológica. A terapia farmacológica se baseia nas classes dos antipsicóticos atípicos e é direcionado de acordo com o tipo de delirium, como exemplo temos o haloperidol, risperidona e quetiapina. Na terapia não farmacológica deve-se afastar do paciente e controlar os fatores deriscos para que não ocorra a problemática.

De acordo com Bastos (2020), o ambiente da Unidade de Terapia Intensiva (UTI) sempre foi visto como um lugar frio e relacionado a sofrimento e morte, conspirando para que fatores de riscos no desenvolvimento do delirium fossem notórios e perpetuando até os dias atuais, afirmando ainda que cerca de $90 \%$ dos pacientes internados na UTI desenvolvem delirium.

Este tema foi escolhido, devido a vivência das pesquisadoras no estágio curricular obrigatório de enfermagem em unidade de terapia intensiva, ao observar um paciente diagnosticado com delirium. Com isso, surgiu a necessidade de entender quais eram os fatoresde riscos que poderiam levar ao paciente desenvolver o delirium na unidade de terapia intensiva com intuito de evitar ou até mesmo intervir a problemática após o surgimento da mesma.

O presente estudo torna - se relevante devido a percepção de que o delirium é algoque sempre esteve presente na UTI e que pode se apresentar em cenários distintos. Sendo assim,este estudo objetiva identificar quais os fatores de riscos intrínsecos 
e extrínsecos que predispõe o delirium em pacientes internados na unidade de terapia intensiva afim de impedir o desenvolvimento do mesmo.

Baseados nas pesquisas existentes, esta pesquisa busca responder a seguinte pergunta norteadora: quais os fatores de riscos intrínsecos extrínsecos que predispõe o deliriumem pacientes internados na UTI?

\section{Metodologia}

Como metodologia, optou - se por trabalhar com a revisão integrativa. As revisõesintegrativas de literatura permitem sintetizar e discutir achados sobre determinado tema estudado. Nesta perspectiva, os estudos de revisão consistem em organizar, esclarecer e resumiras principais obras existentes, bem como fornecer citações completas abrangendo o espectro de literatura relevante em uma área (Casarin, 2020).

A primeira fase da revisão de literatura consiste na identificação do tema e perguntanorteadora, no qual foi utilizado a seguinte pergunta norteadora: quais os fatores de riscos intrínsecos e extrínsecos que predispõe o delirium em pacientes internados na unidade de terapia intensiva?

Em seguida, na segunda fase, foi estabelecido os critérios de inclusão e exclusão eseleção das publicações, em que optou - se por estudos publicados nos anos de 2016 a 2020, realizados em unidade de terapia intensiva e que tratavam da temática delirium. Todos no idioma português, disponíveis na íntegra na base de dados Scientific Electronic Library Online (SCIELO) e (LILACS), a partir de três terminologias em saúde consultadas nos Descritores em Ciências da Saúde (DeCS): "fatores de riscos", "delirium" e "unidade de terapia intensiva". Foram excluídos deste estudo pesquisas que não estavam dentro da temática, os artigos duplicados, os que estavam fora do tempo determinado e que não estavam escritos no idioma português. Inicialmente, foram levantados 39 artigos nas duas bases de dados, no qual encontrou - se 20 na SCIELO e 19 na LILACS.

$\mathrm{Na}$ terceira fase realizou-se a identificação dos estudos selecionados e pré-selecionados, no qual foi realizado leitura do resumo de 13 artigos da SCIELO e 11 da LILACS para avaliar a pertinência ou não em relação à questão norteadora, a seleção dos estudos pertinentes, a organização dos estudos pré-selecionados, identificação por meio de instrumento de avaliação.

A quarta fase foi a categorização dos estudos selecionados, onde fez - se uma análise crítica dos estudos, formação de uma biblioteca individual com os artigos selecionados, elaboração e uso da matriz de síntese; análise das informações; uso dos critérios de validação para a análise crítica dos artigos e conteúdos selecionados; categorização dos conteúdos analisados e que respondem à pergunta clínica de pesquisa.

Em seguida, foi realizada a quinta fase, na qual consiste na leitura dos artigos selecionados na íntegra e a interpretação, no qual foram lidos na íntegra 4 estudos da SCIELO e 3 LILACS. Extraiu-se trechos dos estudos que respondiam à questão norteadora, elegendo-ospara a próxima etapa.

Em seguida, a sexta e última fase, foi elaborado um documento que descreveu detalhadamente a revisão integrativa realizada, além de exposição de propostas para estudos futuros. Para melhor entendimento, elaborou-se um fluxograma da construção do estudo, descrito na Figura 1. 
Figura 1 - Fluxograma de construção do corpus de estudos.

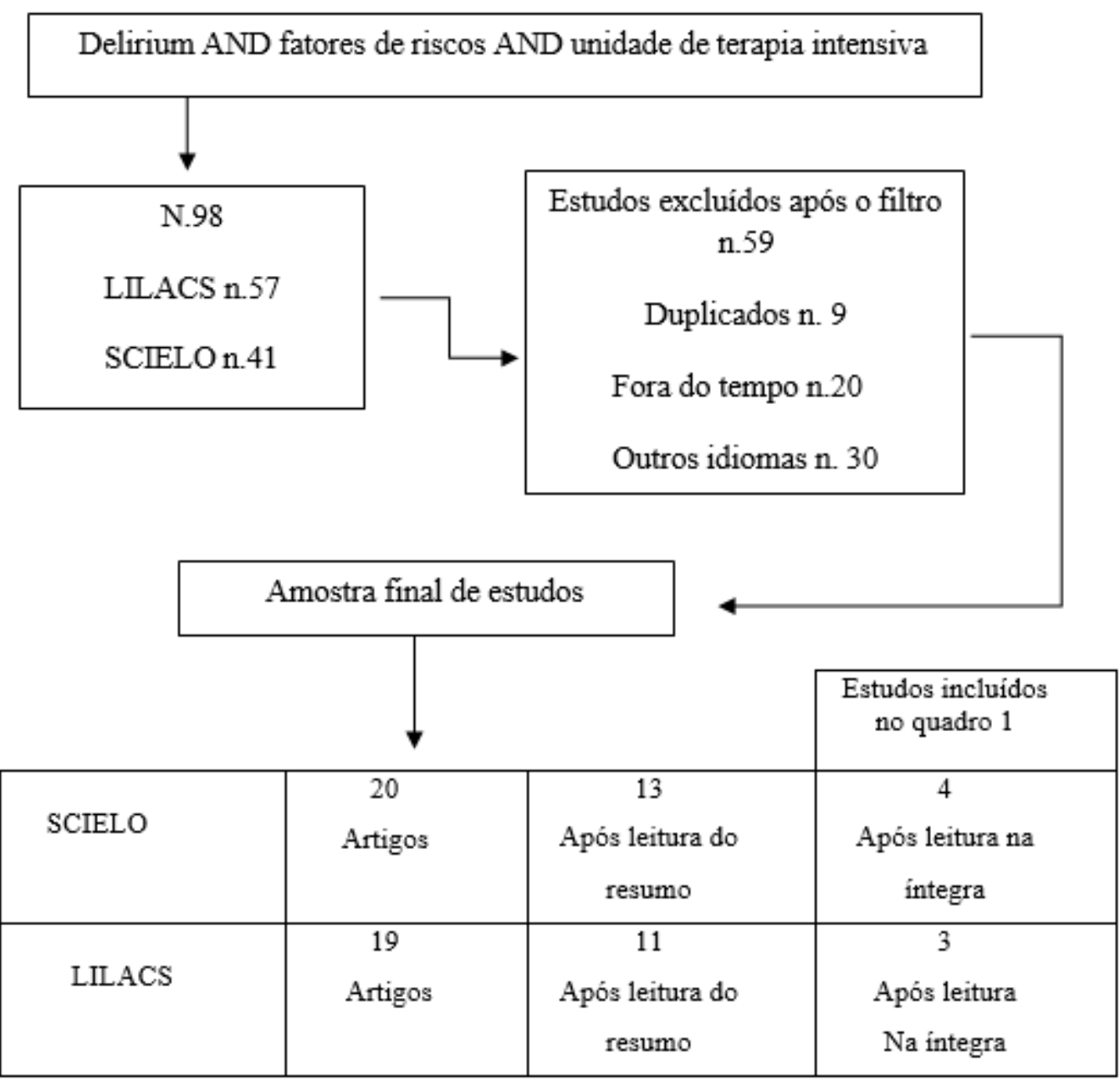

Fonte: Autores.

Destaca - se ainda, que todas as etapas descritas na metodologia, foram seguidasde forma rigorosa pelos autores, para então obter resultados exatos e fidedignos durante a avaliação e construção da discussão.

\section{Resultados e Discussão}

No contexto do delirium, esta revisão integrativa pode deixar claro que a maioria dos estudos encontrados tem o objetivo de correlacionar o aparecimento do delirium com a internação na unidade de terapia intensiva. sobre os artigos incluídos nesta revisão, observou- se que houve uma prevalência de estudos publicados nos anos de 2016 e 2020.

Com relação aos principais fatores intrínsecos e extrínsecos que predispõem o surgimento de delirium na unidade de terapia intensiva, foram elencados os que mais foram citados nas publicações.

As análises sobre a incidência de delirium na UTI indicam que esta pode variar de acordo com as características dos pacientes e de outros fatores. Como resultados, após a análise significativa do conteúdo, obtivemos os seguintes fatores que predispõe; idade igual ou maior que 60 anos, o uso de sedoanalgésicos, ventilação mecânica invasiva, restrição física, desidratação e desnutrição, ambiente típico de uma UTI e perturbação do sono, todos descritos no Quadro 1, onde estão descritos nome do autor, ano de publicação, título do artigo e fatoresde riscos predisponentes. 
Quadro 1 - Identificação dos principais fatores intrínsecos e extrínsecos para o delirium em Unidade de Terapia Intensiva.

\begin{tabular}{|c|c|c|c|c|}
\hline $\mathbf{N}^{\mathbf{o}}$ & $\begin{array}{l}\text { BASE DE } \\
\text { DADOS }\end{array}$ & TÍTULO DO ARTIGO & $\begin{array}{l}\text { AUTORES E } \\
\text { ANO }\end{array}$ & FATOR DE PREDISPOSIÇÃO \\
\hline E1 & Lilacs & $\begin{array}{l}\text { Delirium na terapia intensiva: fatores } \\
\text { predisponentes e prevenção de eventos } \\
\text { adversos }\end{array}$ & $\begin{array}{l}\text { Da Silva et al., } \\
2018 \text {. }\end{array}$ & Idade igual ou maior que60 anos \\
\hline E2 & SciELO & $\begin{array}{l}\text { A influência do delirium no tempo de } \\
\text { ventilação mecânica em pacientes } \\
\text { críticos }\end{array}$ & Krebs et al., 2018. & Ventilação mecânicainvasiva \\
\hline E3 & SciELO & $\begin{array}{l}\text { Incidência e fatores relacionados ao } \\
\text { delirium em unidade de terapia intensiva }\end{array}$ & Mori., 2016. & $\begin{array}{l}\text { Restrição física associada a gravidade } \\
\text { dopaciente }\end{array}$ \\
\hline E4 & SciELO & $\begin{array}{l}\text { Delirium no doente crítico: fatores de } \\
\text { riscos modificáveis pelos enfermeiros }\end{array}$ & Pereira et al., 2016. & Desnutrição edesidratação \\
\hline E5 & LILACS & $\begin{array}{l}\text { O manejo não farmacológico do } \\
\text { delirium sob a ótica de } \\
\text { enfermeiros de uma unidade de terapia } \\
\text { intensiva }\end{array}$ & Eberle et al., 2019. & Pertubação do sono \\
\hline E6 & LILACS & $\begin{array}{l}\text { Assistência de enfermagem ao paciente } \\
\text { com delirium na unidade de terapia } \\
\text { intensiva }\end{array}$ & Gois et al., 2019. & Ambiente típico de umaUTI \\
\hline E7 & SciELO & $\begin{array}{l}\text { Prevalência de delirium em pacientes de } \\
\text { terapia intensiva e associação com } \\
\text { sedoanalgesia, gravidade e mortalidade }\end{array}$ & Bastos et al., 2020. & Sedoanalgesia \\
\hline
\end{tabular}

Fonte: Autores.

A UTI é o local designado para pacientes gravemente enfermos, onde se oferece cuidados contínuos aos mesmos. A internação na UTI pode ser considerada um evento de vidadesgastante, tendo em vista que a admissão é geralmente em função de uma condição crítica. Essas unidades de tratamento são consideradas invasivas e estressoras pelos pacientes. Esta situação, associada às debilidades físicas desses pacientes e à alta complexidade de situações eeventos faz com que o delirium possa estar presente devido aos fatores que elevam o risco desteproblema na unidade de terapia intensiva (Gois, 2019).

Neste estudo, encontrou-se associação do delirium com diversos fatores de riscos para o desenvolvimento do mesmo. No qual Da Silva et al., (2018), diz que a maior ocorrênciade delirium, foi em pacientes com 60 anos, associado a deteriorização cognitiva e suscetibilidade devido alterações fisiológicas do envelhecimento como dificuldade com a memória, tarefas mentais com relação ao raciocínio, essas alterações podem variar em intensidade e dificultam a realização de atividades cotidianas.

Nesse sentido, segundo o autor, esses pacientes são pré dispostos a ocorrência de delirium, entretanto, esse fator não exclui a ocorrência da patologia em pacientes jovens, pois o delirium está relacionado a diversos fatores e não apenas a idade (da Silva et al., 2018), tais como: uso de ventilação mecânica, restrição física, desnutrição e desidratação, privação do sono, influência do ambiente e sedoanalgesia que são os fatores apontados pelos estudos analisados.

Comparando o estudo acima com a coorte de Mesa et al., (2017), podemos perceberque existe uma pequena variação na idade, pois no seu estudo os pacientes que desenvolveram delirium foram os que tinham idade acima de 65 anos, assim como Gois (2019) retrata na sua pesquisa. O mesmo fala na sua coorte que a idade traz consigo uma variedade de problemáticas que podem ajudar no desenvolvimento do delirium, como baixa imunidade, déficit neurológico e outros 
problemas em idosos. Além disso, a população mais idosa vem crescendo e, consequentemente, também ocorre um aumento na taxa de admissão de pacientes mais velhos à UTI.

Além da idade, o estudo de Krebs et al., (2018), traz também como fator de riscoa ventilação mecânica invasiva, no qual delirium pode se manifestar em até $80 \%$ dos pacientesinternados, o que se repete no estudo feito por Gois (2019) e por Martins (2019). Uma vez que pacientes em uso tubo endotraqueal, as secreções e as assíncronas com o ventilador, impossibilitam que esse paciente consiga se comunicar com a equipe e essa dificuldade, segundo o autor, apresenta-se como um fator de risco para o delirium.

Porém, no estudo de Bastos (2016) traz uma diferença, afirmando que tanto os pacientes em ventilação mecânica invasiva quanto os que estavam respirando de forma espontânea desenvolveram delirium, sendo que os que estavam em VM foram um total de 60\%,mostrando uma diferença significativa em relação aos dados de Da Silva et al., (2018), que relata o delirium em apenas 50\% dos pacientes em ventilação mecânica invasiva.

Se tratando do uso de restrição física como um fator de risco para delirium, o autorMori (2016) supõe que aumenta a chance dos pacientes desenvolverem em até 44,3\%, isso acontece devido o paciente crítico não realizar atividades físicas devido a gravidade de sua condição clínica. Segundo Bahia (2016), no delirium ocorre também uma diminuição de neurotransmissores e, a atividade física, mesmo que mínima, ajudaria no aumento de neurotransmissores no organismo do paciente.

Os pacientes internados em unidade de terapia intensiva, são restritos ao leito e por conta da impossibilidade de locomoção e a inatividade física, esses pacientes podem apresentaragitação e aumento da incidência de delirium, situação que associada aos demais fatores, aumenta significativamente a ocorrência em pacientes em tratamento intensivo (Luz, 2017).

No que se refere a desnutrição e desidratação, Pereira et al. (2016) relata que das 37 observações feitas em seu estudo, o delirium se fez presente após o quadro, no qual sete pacientes estavam em dieta zero e doze em dieta entérica, mostrando que existe um risco aumentado, cerca de $75 \%$ para o desenvolvimento de delirium.

Essa dado evidência que o delirium tem sua incidência aumentada significativamente quando se trata de pacientes com alguma dificuldade alimentar desenvolvida ao longo da internação, essas dificuldades devem ser observadas e acompanhadas pela equipe multidisciplinar, uma vez que o paciente com delirium pode desenvolver dificuldade para se alimentar, ou a ocorrência de eventos adversos como a pneumonia por aspiração, má nutrição e suas sequelas, incluindo óbito por inanição, por exemplo (Pereira et al., 2016).

Erbele et al., (2019) também relata na sua discussão a importância nutrição, salientando que esta pode levar ao delirium, pois a mesma se trata de uma disfunção orgânica e as disfunções orgânicas são fatores de risco para o desenvolvimento de delirium.

Os estudos analisados nesse trabalho, também apontam a privação de sono como um fator de risco para a ocorrência de delirium. Segundo Mori et al., (2016), em virtude dos pacientes internados na Unidade de Terapia Intensiva apresentarem redução da qualidade de sono, fragmentação e outros distúrbios, podem ser acometidos por delirium. O autor contextualiza a análise através da relação da privação de sono em pessoas não acometidas por nenhuma patologia, identificando que a falta de sono provoca desorientação, flutuação da capacidade mental e disfunção cognitiva, essas mesmas características também podem ser percebidas em pacientes com delirium internado na UTI.

O autor Mori et al., em seus estudos de 2016 completa ainda que as modificações ocasionadas pelos distúrbios de sono e de delirium, com relação as estruturas anatômicas e neurotransmissores apresentam semelhança considerável. Além disso, essas duas disfunções serelacionam, podendo o delirium antecipar a privação de sono.

Os pacientes da UTI, além das dificuldades impostas pela suas condições físicas, estão em um ambiente com ruídos, os procedimentos, os bipes de aparelhos e interações entre a equipe de saúde, podem ocasionar a dificuldade no sono, como 
aponta Gois et al., (2019), ospacientes em tratamento na UTI dormem em média apenas duas horas por dia e menos de $6 \%$ do seu sono é do tipo REM (Rapid Eye Movement), condição essa que é considerada como um fator determinante para a incidencia de delirium nesses pacientes.

Erbele et al., (2019) cita em seus estudos, que a perturbação do sono está associadaao delirium, uma vez que prejuízo do sono foi abordado como disparador das disfunções neurológicas, relatando ainda que há uma grande necessidade de qualidade do sono. Souza et al., (2018) esclarece que a perturbação do sono acarreta em desregulação do ciclo circadiano e, possivelmente, prejudica a atenção e a orientação do paciente, potencializando o desenvolvimento de delirium.

No estudo de Da Silva et al., (2018), foi identificado que $27 \%$ dos pacientes com privação do sono apresentaram delirium, ocorrendo uma relação relevante comparado aos outros fatores que predispõe. Sabe-se que a privação do sono é prejudicial à saúde, principalmente daqueles que estão internados, pois necessitam de repouso, podendo diminuir aimunidade, causar problemas neurológicos e respiratórios, prolongar a duração da ventilação e consequentemente o tempo de internação na unidade de terapia intensiva.

Outro fator considerado determinante para a ocorrência de delirium é o próprio ambiente hospitalar, assim como as questões relacionadas aos distúrbios de sono, o ambiente da UTI é um ambiente que é permeada pelo medo para os pacientes, a utilização dos recursos tecnológicos invasivos e por vezes dolorosos, a linguagem técnica, a dependência da equipe desaúde, além do compartilhamento dessa experiência com indivíduos fora do ambiente familiar,são fatores que podem influenciar na ocorrência de delirium em pacientes da unidade de terapiaintensiva (Martins,2019).

As Unidades de Terapias Intensivas são unidades criadas para atendimento de pacientes que necessitam de cuidados diretos e intensivos, pois seu quadro de saúde pode facilmente evoluir para a morte. É considerado um setor de pouco entrosamento com outros setores do hospital bem como há pouco entrosamento entre a equipe e o doente. Tem-se um ambiente, na maioria das vezes, técnico com o cuidado mecanizado com foco no tratamento da doença e não no próprio paciente. Esse contexto, transforma o ambiente hospitalar em um lugarpouco acolhedor para os pacientes e essa situação pode ser considerada como predisposição para a ocorrência de delirium em pacientes que estejam internados (Gois et al., 2019).

Segundo Faustinol et al. (2016), em virtude dos pacientes nesse ambiente hospitalar estarem mantidos sob sedação durante o período de recuperação dos níveis de consciência, pode ocorrer desse pacientes sentirem privações sensoriais capazes de causar alucinações. Nesse contexto, o ambiente da UTI interfere no estado do paciente, pois existe a ausência de luz solar, de relógios, televisão, visitantes e até mesmo outros pacientes, quando os mesmos estão em UTI individual, podendo levar à falta de orientação e espaço do paciente (Gois et al., 2019).

Em comparação a Faustinol et al., (2016), que verificou que a maioria dos problemas identificados pelos participantes contemplou fatores de risco para desenvolvimentodo delirium e estavam relacionados ao ambiente da UTI como um todo, chamando atenção emseu estudo para os ruídos e a iluminação excessiva também.

No estudo de Souza et al., (2018), evidenciou-se que a ocorrência de delirium empacientes da UTI ocorrem quando esses estão em ambiente sem a presença de outros pacientes, ou seja, o isolamento também configura-se como um fator determinante. O quadro se estendea paciente que não recebem visita ou que mudam de ambiente repentinamente. Além disso, a ausência de luminosidade natural nesse ambiente hospitalar possibilita é também um fator considerado na análise sobre o delirium em pacientes críticos.

Se tratando da sedoanalgesia, Bastos et al., (2020) relata que os pacientes de seu estudo, fizeram pelo menos o uso de um fármaco, e que os mesmos tiveram grande associaçãocom o delirium, sendo o fentanil, em primeiro lugar com 43,3\%, midazolan em segundo com $36,9 \%$, em terceiro propofol $14 \%$ e quarto e último a clonidina $12,7 \%$. 
Em comparação ao estudo de da Silva et al., (2018), que afirma a relação de sedoanalgesia com o delirium, chegando a acometer, um total de 39\% dos pacientes de sua pesquisa que estavam na UTI. Além disso, trouxe a morfina, midazolam, lorazepam, diazepam,propofol, meriperidona, atropina e hioscina e fentanil como os mais associados a delirium.

O autor completa que o fentanil foi o que esteve com maior associação ao deliriumem sua pesquisa (SILVA et al. 2018), se diferenciando da pesquisa de Bastos et al., (2018) descrito no penúltimo parágrafo, que associou o fentanil como um dos principais sedoanalgésicos que predispõe ao delirium dentro da UTI.

Segundo Bastos et al., (2020), existem fatores que precipitantes do delirium, o autor aponta além das medicações que possuem potencial psicoativo conhecido como os sedativo-hipnóticas que aumentam o risco de delirium de 3 a 12 vezes, os narcóticos que aumentam em 3 vezes e os anticolinérgicos que aumentam de 5 a 12 vezes a ocorrência de delirium. Além disso, fatores ambientais e psicossociais, infecções ocultas, falência respiratória, são considerados por esse autor como fatores que podem influenciar na incidência de delirium.

Nesse contexto, os sintomas de delirium são comuns em pacientes internados na UTI em uso de sedoanalgesia e devem ser investigados, pois é um problema que não possui tratamento farmacológico bem definido, e que influência diretamente no prognóstico (Silva et al. 2018).

Desse modo, a Unidade de Terapia Intensiva (UTI) é tida como um local onde se presta assistência qualificada especializada, independentemente de os mecanismos tecnológicosutilizados serem cada vez mais avançados, capazes de tornar mais eficiente o cuidado ao paciente em estado crítico, e necessário o controle efetivo desses pacientes, uma vez que o delirium pode ocorrer com significativa incidência a partir dos fatores relacionados apontados como predeterminante para pacientes em tratamento intensivo.

Esses fatores são constituídos e identificados a partir de um conjunto de elementosfuncionalmente agrupados, que foram apresentados nos estudos analisados e que apontam paraa necessidade de qualificação e capacitação dos profissionais da saúde para acompanhamento desses pacientes quando acometidos por delirium.

\section{Considerações Finais}

Diante deste estudo, podemos afirmar que o delirium é um problema muito comumem pacientes internados em UTI, podendo influenciar no prognóstico e a qualidade de vida dopaciente internado em unidade de terapia intensiva.

O primeiro passo para intervir nessa situação é prevenir, criando protocolos de prevenção de delirium baseado nos fatores de risco com intuito de chamar a atenção dos profissionais para os pacientes que apresentarem um resultado positivo para esses fatores.

Além disso, o delirium deve ser considerado uma emergência na UTI, e educar os profissionais quanto a isso é de extrema importância, para que os mesmos fiquem em constantevigilância neurológica para que possam agir de forma assertiva no diagnóstico e tratamento domesmo.

Essas mudanças relacionadas ao desenvolvimento do conhecimento da equipe de saúde se fazem necessária para uma reflexão das ações realizadas no cotidiano, e, consequentemente, mais preparo dos profissionais, não só sob o aspecto teórico e técnico, relacionados a ocorrência de delirium, mas, também, voltada à transformação da assistência numa perspectiva mais humanitária.

Desse modo, a pesquisa teve como propósito responder a problemática sobre quais os fatores de riscos intrínsecos extrínsecos que predispõe o delirium em pacientes internados na UTI, apresentando como principais fatores: idade igual ou maior que 60 anos, o uso de sedoanalgésicos, ventilação mecânica invasiva, restrição física, desidratação e desnutrição, ambiente típico de uma UTI e perturbação do sono. Assim, esse estudo atendeu sua finalidadede modo eficiente e satisfatório. 
Ainda existe poucos dados sobre a temática, sugere-se pesquisas futuras para melhor entendimento do problema, como entender o perfil dos pacientes diagnosticados com delirium internados em UTI e buscar entender se o delirium é um distúrbio que acontece apenas dentro do ambiente da UTI, além de tentar entender quais mecanismos fisiopatológicos estão envolvidos, visto que quase não há estudos sobre estes.

\section{Agradecimentos}

\section{Joyce Pereira Santos}

Agradeço em primeiro lugar, a Jeová Deus, que me deu saúde, determinação e perseverança para lutar pelos meus objetivos. Agradeço aos meus pais; Adimilde Maria Pereira e José Ribamar da Paixão Santos por me ensinarem o caminho correto e por sempre estarem me apoiando ao longo de toda a minha vida em todos os passos que dei. A minha família, em especial aos meus irmãos que eu tanto amo (Josemilde, Jeane Fernanda, Rondinelly, Gerdson, Herbert), minhas tias (Eliane Cristina Pereira, Conceição de Maria Pereira e a Leuzilene de Jesus Santos Cruz), ao meu tio Durvã por ter me dado o meu primeiro sapato branco com toda boa vontade do mundo e pelas seguintes palavras: "Gente pra te dizer que teu curso não irá prestar terá muitas, mas você tem que tampar os ouvidos para essas pessoas e seguir em frente". Agradeço também ao meu tio postiço Pedro Agnaldo Brenha que me ajudou de várias formas durante as etapas da minha vida e da graduação. Aos meus padrinhos (Luís Henrique e Palmelita) por todo incentivo aos estudos desde criança e pelo apoio financeiro em toda minha vida escolar e acadêmica, me dando dinheiro para passagem, material escolar, mensalidades e publicações. A minha amiga Nayara Martins e sua família (Maria e Aldely), por sempre me acolherem quando mais precisei. A Jorge Andrade por ter me ajudado de uma forma tão carinhosa e com muito boa vontade. Agradeço também ao meu grande amor, Milton César da Conceição Sousa, que apesar de ter chegado no final de minha trajetória quanto acadêmica, me incentivou e me incentiva de várias formas para que eu continue nessa caminhada e torce pelo meu sucesso. A minha orientadora Kássia Cristhine Nogueira Gusmão por aceitar conduzir minha pesquisa. Aos meus professores de graduação, que me conduziram em ótimos caminhos na faculdade e me inseriram em diversas atividades, onde pude crescer como acadêmica (Rafael Mondego e Vera Lopes Barros). Agradeço do fundo do meu coração a Dra. Maria Cristiane Aranha Brito, por todos ensinamentos e convites para pesquisas e por me direcionar. Agradeço muito a minha coordenadora de curso Rose Daiana Cunha dos Santos, pelos puxões de orelha e por sempre me enxergar como uma aluna esforçada e me lançar várias propostas e oportunidades que me levaram a lugares incríveis. Meu muito obrigada a todos pelas palavras boas e de incentivo, quero que saibam que isso foi de extrema importância para que eu continuasse, pois sem vocês eu não conseguiria, toda a contribuição de vocês eu jamais esquecerei por toda a minha vida. Desejo que Deus ilumine a todos e que vocês continuem sempre esses incentivadores de pessoas, isso toca pessoas e move o mundo. Amo muito todos vocês. Serei eternamente grata e nem sei como retribuí-los.

\section{Nayara Martins Pestana Sousa}

Ser enfermeira é um sonho e esse sonho está prestes a ser realizado e tenho muito a agradecer às pessoas que foram essenciais nessa trajetória. Agradeço à Deus em primeiro lugar por me dar saúde, fé, perseverança, por ter iluminado o meu caminho e por me ajudar a ultrapassar os obstáculos encontrados durante a minha vida, principalmente acadêmica. Sou grata aos meus pais Aldely e Nelson que nunca desistiram de mim e me ajudaram a embarcar nesse sonho. Ao meu irmão Mateus que mesmo longe me apoiou, a minha Avó Maria, aos meus primos Ariane e Ítalo, aos meus padrinhos Rogério e Maria pela força e incentivo. Agradeço ao meu marido e amigo Luiz Carlos que sempre esteve presente nos momentos difíceis com uma palavra de incentivo. Agradeço também ao meu filho Luiz Otávio por ter me inspirado ainda mais a tornar esse sonho realidade. Aos meus amigos, em especial Joyce Pereira pela força, incentivo e por compartilhar momentos incríveis. Agradeço à minha orientadora Kássia Cristhine Nogueira Gusmão por aceitar conduzir esta pesquisa com dedicação. A todos os meus colegas do curso de graduação que compartilharam inúmeras experiências e desafios que enfrentamos ao longo da caminhada. Também agradecer à Instituição e a minha coordenadora do curso Rose Daiana Cunha dos Santos por sempre incentivar e me dar bons conselhos, aos professores por me darem a ferramentas para evoluir essencialmente no meu processo de formação profissional, pela dedicação, e por tudo o que aprendi ao longo dos anos do curso. O meu muito obrigada a todos que de forma direita ou indireta fazem parte desse sonho. 
Research, Society and Development, v. 10, n. 13, e166101321072, 202

(CC BY 4.0) | ISSN 2525-3409 | DOI: http://dx.doi.org/10.33448/rsd-v10i13.21072

\section{Referências}

Bahia, A. A. (2016). Delirium No Paciente CTI. Repositório da Universidade Federal De Minas Gerais, Minas Gerais.

Bastos, A. S., Beccaria, L. M., Silva, D. C. \& Barbosa, T. P. (2020). Prevalência de Delirium em pacientes de Terapia Intensiva e associação com sedoanalgesia, gravidade e mortalidade. Revista Gaúcha de Enfermagem, 41(4), 123-137.

Casarin, S. T., Porto, A. R., Gabatz, R. I. B., Bonow, C. A., Ribeiro, J. P. \& Mota, M. S. (2020). tipos de revisão de literatura: considerações das editoras do Journal of Nursing. Journal of Nursing and Health, 10(5).

Da Silva, M. H. O., Camerini, F. G., Henrique, D. M., Almeida, L. F., Franco, A. S. \& Pereira, S. R. M. (2018). Delirium Na Terapia Intensiva: Fatores Predisponentes E Prevenção De Eventos Adversos. Revista Baiana De Enfermagem, 32(3).

Eberle, C. C., Santos, A. A., Macedo Júnior, L. J. J. \& Juliana Bessa Martins. (2019) O manejo não farmacológico do delirium sob a ótica de enfermeiros de uma Unidade de Terapia Intensiva adulto. Revista de Pesquisa: cuidado é fundamental online, 11(5), 1242-1249.

Faustinol, T. N., Pedreiral, L. C., Freitas, Y. S., Silva, M. O. \& Amaral, J. B. (2016). Prevenção e monitorização do delirium no idoso: uma intervenção educativa. Revista Brasileira de Enfermagem, Rio de Janeiro, 4(69), 725-32.

Gati, J. P. S. (2017). Aspectos Linguísticos Do Discurso Delirante. Instituto de estudo da linguagem da Universidade Estadual de Campinas, São Paulo.

Gois, J., Vieira, T., Lourenço, B. S., Sousa, D. R. S., Valenin, L. \& Reis. L. (2019). Assistência De Enfermagem Ao Paciente Com Delirium Na Unidade De TerapiaIntensiva: Uma Revisão Integrativa. Revista Nursing, 22(23), 3214-3219.

Krebs, J. A., Osaku, J. F., Costa, C. R. L. M., Ogasawara, S. M., Costa, J. B., Taba, S., Jorge, A. C. \& Duarte, P. D. D. (2018). a influência do delirium no tempo de ventilação mecânica em pacientes críticos: uma revisão sistemática. Arquivos Brasileiros de Ciências da Saúde, (43), 61-66.

Mori, S., TakedaJ. R. T., Carrara, F. S. A., Cohrs, C. R., Zanei, S. S. V. \& Whitaker, I. Y. (2016). incidence and factors related to delirium in an Intensive Care Unit. Revista da escola de Enfermagem da USP, 50(4), 587-593.

Lima, M. L. M. N., Moreira, R. O., Okamoto, O. T. O. \& Amaral, J. B. (2016). Delirium Em Terapia Intensiva: Revisão Sistemática. Repositório Institucional

Escola Bahiana de Medicina e Saúde Pública, Bahia.

Luz, L. F. \& Manozzo, B. M. (2016). Associação do delirium com cognição,capacidade funcional e qualidade de vida no paciente crítico. SEFIC.

Luz, L. F. D. S., Santos, M. C. D., Ramos, T. A., Almeida, C. B. D., Rover, M. C., Dal'Pizzol, C. P. \& Boniatti, M. M. (2020). Delirium e qualidade de vida em pacientes críticos: um estudo de coorte prospectivo. Revista Brasileira de Terapia Intensiva, 32, 426-432.

Martins, J. B., dos Santos, A. A., de Macedo Júnior, L. J. J., \& Eberle, C. C. (2019). Avaliação da prevalência de delirium em uma unidade de terapia intensiva pública. Enfermagem em Foco, 10(3).

Mesa, P., Previgliano, I. J., Altez, S., Favretto, S., Orellano, M., Lecor, C. \& Ely, E. (2017). Delirium em uma unidade de terapia intensiva latino-americana. Estudo prospectivo em coorte em pacientes em ventilação mecânica. Revista Brasileira de Terapia Intensiva, 29, 337-345.

Pereira, J. M., Barradas, F. J. D. R., Sequeira, R. M. C., Marques, M. D. C. M. P., Batista, M. J., Galhardas, M., \& Santos, M. S. (2016). Delírium no doente crítico: fatores de risco modificáveis pelos enfermeiros. Revista de Enfermagem Referência, 4(9), 29-36.

Pereira, M. A. (2019). Modelagem dos processos de monitoramento do delirium utilizando o fluxograma do CAM-ICU: prototipagem de plataforma digital para atendimento ao paciente crítico.

Prayce, R., Quaresma, F., \& Neto, I. G. (2018). Delirium: The 7th Vital Sign? Acta medica portuguesa, 31(1), 51-58.

Santos, F.S. Mecanismos fisiopatológicos do delirium. (2005). Revista. Psiquiatria, 3(32), 104-112.

Sosa, F. A., Roberti, J., Franco, M. T., Kleinert, M. M., Patrón, A. R., \& Osatnik, J. (2018). Avaliação de delirium com uso do modelo PRE-DELIRIC em uma unidade de terapia intensiva na Argentina. Revista Brasileira de Terapia Intensiva, 30, 50-56.

Souza, T. L., Azzolin, K. O. \& Fernandes, V. R. (2018). Cuidados multiprofissionais para pacientes em delirium em terapia intensiva: revisão integrativa. Revista Gaúcha de Enfermagem, 39(7).

Veiga, V. C. \& Rojas, S. S. O. (2018). Analgossedação e Delirium em Unidades de Terapia Intensiva Brasileiras: Como Estamos na Atualidade. Estudo ASDUTI. Revista Brasileira de Terapia Intensiva, 30(2), 246-8. 